DOI: $10.3901 / J M E .2014 .19 .151$

\title{
生物凝胶微孔挤出胀大的有限元 模拟与三维打印成形"
}

\author{
于永泽陈海萍 胡庆夕 \\ (上海大学快速制造工程中心 上海 200444)
}

\begin{abstract}
摘要: 由于挤出胀大现象的存在, 在室温条件下通过三维打印技术(Three-dimensional printing, 3DP)成形的凝胶组织工程支 架与输入的目标支架原始模型相比, 内部微观孔隙结构精度与孔隙率明显降低。针对这一问题, 采用流体计算软件 Polyflow 对明胶-海藻酸钠共混体系交联形成的生物凝胶材料在挤出成形过程中通过喷头直径过渡段时流线的收缩与离开喷头后的挤 出胀大现象进行有限元模拟, 着重分析凝胶秥度与供料压力对挤出胀大的影响规律, 并通过试验验证模拟的合理性。提出将 挤出胀大引起的尺寸变形误差引入支架模型的设计过程。通过数值模拟与试验结果的对比, 合理调整模型中纤维间距, 利用 得到的最佳工艺参数进行凝胶组织工程支架的三维打印成形。结果表明, 支架内部的微观孔隙结构精度和孔隙率与理想支架 要求近似吻合。通过对生物凝胶挤出胀大的模拟分析, 可为凝胶组织工程支架的精确打印成形提供理论依据和技术指导。
\end{abstract}

关键词: 凝胶支架; 三维打印; 挤出胀大; 有限元法; 孔隙率

中图分类号: TH164

\section{Finite Element Simulation of Bio-gel Micropore Extrudate Swell and Three-dimensional Printing Manufacturing}

\author{
YU Yongze CHEN Haiping HU Qingxi
}

(Rapid Manufacturing Engineering Center, Shanghai University, Shanghai 200444)

\begin{abstract}
Due to the extrudate swell effect, the accuracy of porous structure and porosity of the gel scaffold, which is fabricated by three-dimensional printing (3DP) at room temperature, are significantly lower than that of the designed scaffold model. To solve this problem, the CFD package Polyflow is used to simulate the streamline shrinkage and extrudate swell of bio-gel material flowing through the injecting nozzle. The influences of the viscosity and applied pressure on the extrudate swell are discussed, and the reliability of the simulation is verified by experiment. The bio-gel material is cross-linked by gelatin and sodium alginate. The compensation of the material size deformation in the forming process is considered in the design of scaffold model. According to comparison between the numerical simulation and the experimental results, the fiber spacing of the scaffold model is reasonably adjusted, and the gel scaffold is fabricated by utilizing the optimized process parameters. The results show that the accuracy of porous structure and porosity of the gel scaffold are proximate to the ideal scaffold. All the above work can provide the theoretical basis and technical guidance for the gel scaffold 3D printed process accurate control.
\end{abstract}

Key words: gel scaffold; three-dimensional printing; extrudate swell; finite element method; porosity

\section{0 前言}

作为细胞三维受控组装成形的重要载体, 在 $0 \sim 37{ }^{\circ} \mathrm{C}$ 条件下利用凝胶类生物材料制备的组织工 程支架为种子细胞的增殖和分化提供生存空间, 为 细胞获取氧气、营养物质和新陈代谢提供通道, 为 封装的细胞提供保护和支撑并决定再生组织器官的

* 国家自然科学基金资助项目(51075253，51375292)。20131029 收到初 稿, 20140701 收到修改稿
结构形态和尺寸大小。因此，凝胶支架的结构特点、 力学性能和生物理化性质对体外构建含有细胞的活 性组织或器官具有重要的影响。特别是支架中精确 的微观孔隙结构和高孔隙率对种子细胞的形态和基 因表达起着决定性的作用 ${ }^{[1-2]}$ 。微观孔隙结构的形 状、大小、连通性和均匀性直接影响细胞与支架间 的交互作用，细胞特异性基因的表达以及支架的降 解速度是否均衡。较高的孔隙率和比表面积则有利 于细胞外基质的分泌、沉积, 营养物质的输送和代 谢废物的排出，促进新生血管和神经的长 $\lambda^{[3-4]}$ 。 
基于计算机辅助设计和离散-堆积原理, 三维打 印技术(Three-dimensional printing, 3DP)以其高柔性 的数字化驱动加工方式被广泛应用于凝胶组织工程 支架的制备。为了研究材料特性和工艺参数对支架 精确打印成形的影响规律, 国内外学者进行了大量 的研究和试验工作。VOZZI 等 ${ }^{[5-6]}$ 利用 HagenPoiseuille 定律建立了生物材料在喷头内流动的牛 顿流体模型。KHALIL 等 ${ }^{[7-8]}$ 根据流变学理论研究凝 胶材料的非牛顿流体性质对支架打印成形过程的影 响。ALEXE-LONESCU 等 ${ }^{[9-10]}$ 在非牛顿流体模型基 础上研究凝胶材料与喷头壁面之间的滑移和剪切变 稀对支架孔径和孔隙率的影响。LI 等 ${ }^{[11]}$ 建立了凝胶 的体积流速与支架孔径和孔隙率之间的关系模型, 分析供料速度和喷头的运动速度对支架成形精度的 影响。李生杰等 ${ }^{[12]}$ 对凝胶在挤出成形过程中的流场 压强和弹性变形进行建模计算, 采用分段温控和释 放补偿解决细胞受控组装过程中的响应延迟和流涎 问题。杨敏等 ${ }^{[13]}$ 利用混流喷头系统和脉冲切换控制 电路实现多细胞的精确组装。然而, 这些数值模型 的建立与成形精度的评价都是以支架的纤维直径和 喷嘴内径一致为前提, 忽略了凝胶材料在微孔挤出 成形过程中由于其黏弹性流体性质引起的挤出胀大 对支架微观孔隙结构精度和孔隙率的影响。

基于以上分析, 本文利用有限元模拟方法, 通 过流体计算软件 Polyflow 对生物凝胶材料的微孔挤 出成形过程进行数值模拟: 采用参数渐近法处理黏 弹性 Phan-Thien-Tanner (PTT)本构方程中的非线性 参数, 迭代求解凝胶材料由牛顿流体转向黏弹性流 体过程中通过喷头直径过渡段时的流线分布状态和 流场的变化规律; 使用区域划分和网格重构的方法, 模拟分析材料黏度和供料压力对凝胶材料挤出胀大 的影响规律。将模拟结果与试验结果进行对比, 为 合理设计支架的数字化模型、降低挤出胀大对支架 微观孔隙结构精度和孔隙率的影响提供了可靠的理 论指导与最优的工艺参数。结果表明, 通过对生物 凝胶微孔挤出胀大过程的模拟, 可以为深入理解凝 胶支架精密挤出成形过程及工艺参数的优化设计提 供参考依据。

\section{1 生物凝胶挤出胀大的有限元模拟}

\section{1 理论模型的建立}

假设生物凝胶材料的挤出流动过程为不可压 缩、无壁面滑移的等温层流状态。由于材料的高黏 性, 成形过程中流动速度缓慢, 忽略惯性力和重力 的影响, 可以得到凝胶流体的质量守恒与动量守恒
方程

$$
\begin{gathered}
\nabla \cdot \boldsymbol{u}=0 \\
\nabla \cdot \boldsymbol{\tau}-\nabla p=0
\end{gathered}
$$

式中 $\boldsymbol{u}$ 一流体的速度矢量;

$\boldsymbol{\tau}$ ——黏弹性流体的偏应力张量;

$p$ 一材料静压力。

通常, 可以将偏应力张量分解为弹性分量和黏 性分量, 即

$$
\tau=\tau_{\mathrm{p}}+\boldsymbol{\tau}_{\mathrm{s}}
$$

式中 $\tau_{\mathrm{p}}$ 一偏应力张量的弹性分量;

$\tau_{\mathrm{s}}$ 一偏应力张量的黏性分量。

$$
\boldsymbol{\tau}_{\mathrm{s}}=2 \eta_{\mathrm{s}} \boldsymbol{D}
$$

式中 $\eta_{\mathrm{s}}$ 一零剪切黏度中的黏性分量;

$D$ 一一形变速率张量。

采用 PTT 率弹性本构方程与上述控制方程联 立，模拟凝胶材料的微孔挤出成形过程。

PTT 模型

$$
\exp \left[\frac{\varepsilon \lambda}{\eta_{\mathrm{p}}} \operatorname{tr}\left(\boldsymbol{\tau}_{\mathrm{p}}\right)\right] \boldsymbol{\tau}_{\mathrm{p}}+\lambda\left[\left(1-\frac{\xi}{2}\right) \boldsymbol{\tau}_{\mathrm{p}}+\frac{\xi}{2} \boldsymbol{\tau}_{\mathrm{p}}\right]=2 \eta_{\mathrm{p}} \boldsymbol{D}
$$

式中 $\varepsilon, \xi$ 一与材料拉伸行为和第二法向应力 差相关的参数;

$\lambda$ —松弛时间;

$\eta_{\mathrm{p}}$ —零剪切黏度中的弹性分量;

$\nabla, \Delta$ 一上、下随体导数。

\section{2 有限元模型的建立}

图 1 为凝胶组织工程支架三维打印成形系统中 基于一次性注射器的气动式注射喷头。在支架打印 成形的过程中, 凝胶流体在气压作用下由大直径流 道进入小直径过渡段, 最后通过微尺度针头挤出成 形。根据戴元坎等 ${ }^{[14]}$ 的研究成果, 黏弹性流体在经 过流道的直径收缩段时会受到强烈的拉伸变形，存 储大量的弹性能; 流体在喷头内流动时得到部分的 松弛、回复, 离开喷头后残留的弹性能完全释放, 导致材料的挤出胀大。因此, 将有限元模型分为两 个子模型(子计算区域)：喷头直径过渡段和针头挤 出自由段。根据喷头结构的对称性, 为了提高计算 效率, 采用二维轴对称模型的上半部区域代替整个 流道区域, 对流道过渡段和针头出口处的网格进行 局部加密, 得到如图 2 所示模拟生物凝胶微孔挤出 胀大的有限元模型。

\section{3 边界条件与参数设置}

入口边界条件: 凝胶流体在进入流道过渡段之 前为充分发展的流动, 初始体积流量 $Q=\mu S \sqrt{2 \Delta p / \rho}$ 。其中, 流量系数 $\mu=0.6$, 挤出过 


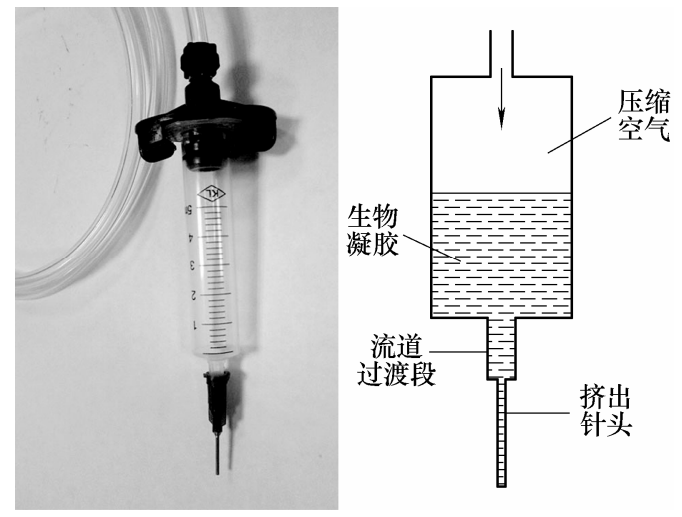

图 1 气动式注射喷头

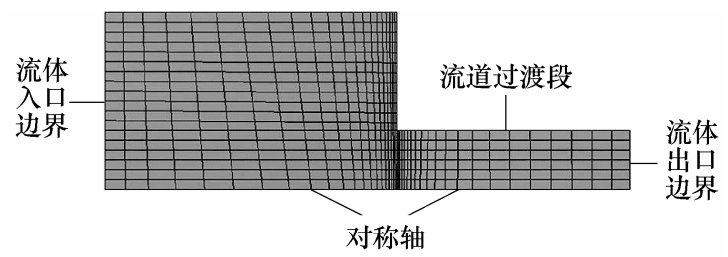

(a) 喷头直径过渡段有限元模型

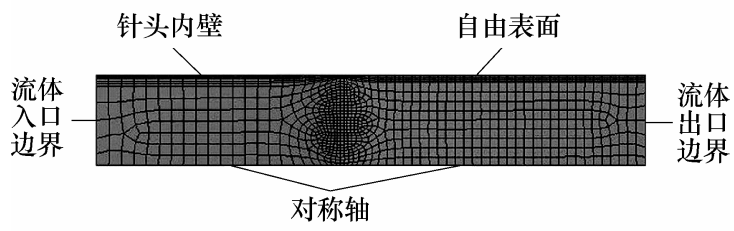

(b) 针头挤出自由段有限元模型

图 2 生物凝胶微孔挤出胀大的有限元模型

程中的压力降 $\Delta p=p_{0}-2 \sigma / d-p_{\mathrm{a}}{ }^{[15]}, p_{0}$ 为初始供 料压力, $p_{\mathrm{a}}$ 为大气压, 流体表面张力参数 $\sigma=35 \mathrm{~Pa} / \mathrm{s}$, 针头内径 $d=0.5 \mathrm{~mm}$; 将流体通过流 道过渡段出口时的模拟体积流量作为流体进入针头 挤出自由段的入口流量。

流道内壁边界条件：设定流道壁面为静止、无 滑移状态, 即 $u_{\mathrm{n}}=u_{\mathrm{s}}=0$ 。

出口边界条件: 流体由针头出口挤出后, 所受 到的法向力和切向力为零, 即 $f_{\mathrm{n}}=f_{\mathrm{s}}=0$ 。

自由表面边界条件: 流体的速度场与自由表面 相切, 即 $f_{\mathrm{n}}=0, u_{\mathrm{n}}=0$; 对自由表面边界的网格 采用网格重构的方法来调整自由表面因挤出胀大而 发生变形的网格。

对称轴边界条件: 忽略所有变量, 设置流体的 切向应力和法向流速为零, 即 $f_{\mathrm{s}}=0, u_{\mathrm{n}}=0$ 。

上述条件中, $f$ 和 $u$ 分别代表应力和速度, $\mathrm{n}$ 和 $\mathrm{s}$ 分别代表法向和切向。

材料选择质量分数比为 $3: 2$ 的明胶、海藻酸钠 共混 ${ }^{[16]}$ 体系在 $4{ }^{\circ} \mathrm{C}$ 条件下交联形成的生物凝胶, 密 度为 $1056 \mathrm{~kg} / \mathrm{m}^{3}$, 松弛时间 $\lambda=1 \mathrm{~s}$, PTT 模型中的 材料参数 $\varepsilon$ 和 $\xi$ 分别取 $0.015 、 0.15, \eta_{\mathrm{s}} / \eta=1 / 9$ 。

\section{2 模拟结果与讨论}

\section{1 喷头直径过渡段的流场模拟结果}

设定初始供料压力 $p_{0}=0.5 \mathrm{MPa}$, 材料黏度 $\eta=1 \mathrm{~Pa} \cdot \mathrm{s}$ 。将渐近函数: $f(S)=S(0 \leqslant S \leqslant 5)$ 施加于 材料的松弛时间，利用迭代分步算法模拟生物凝胶 材料在牛顿流体状态和黏弹性流体状态下通过喷头 直径过渡段时的流线分布特点。不同松弛时间下凝 胶流体通过喷头直径过渡段的等值线流体形态如图 3 所示: 当凝胶材料在喷头中流动时，由于过渡段 流道横截面积突然收缩, 流体受到剧烈的拉伸和剪 切作用，产生非均匀流动，在喷头过渡段的拐角处 形成局部环流; 随着松弛时间的增加，材料由牛顿 流体向黏弹性流体过渡后，流体在喷头过渡段拐角 处的局部环流现象逐渐加剧。在凝胶组织工程支架 打印成形过程中，局部环流会导致材料长时间停留 在喷头内, 引起材料的分解或封装细胞的死亡。因 此，在保证凝胶支架成形质量的前提下应尽量降低 材料的黏度，避免环流作用的出现。
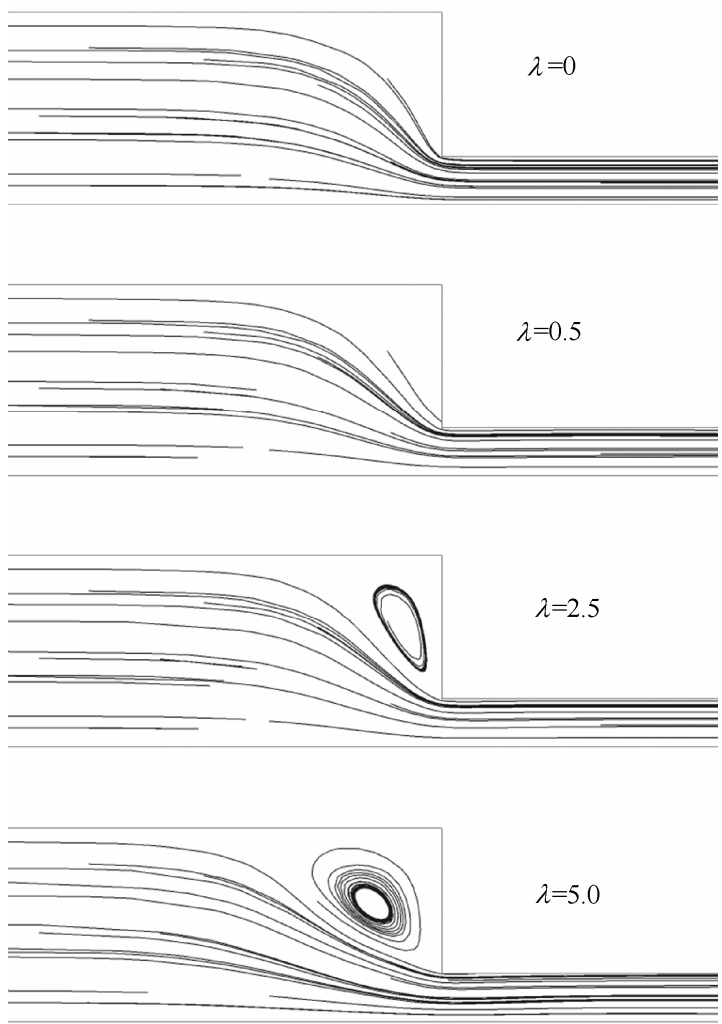

图 3 喷头直径过渡段不同松弛时间的流体形态

\section{2 工艺参数对生物凝胶挤出胀大的影响}

黏度是反映材料流变性能的重要参数, 不同的 黏度值直接影响凝胶材料在流道内的流动阻力和速 度梯度。取初始供料压力 $p_{0}=0.5 \mathrm{MPa}$, 材料黏度 $\eta$ 分别为 $0.05 \mathrm{~Pa} \cdot \mathrm{s} 、 0.5 \mathrm{~Pa} \cdot \mathrm{s} 、 1 \mathrm{~Pa} \cdot \mathrm{s} 、 5 \mathrm{~Pa} \cdot \mathrm{s} 、 20 \mathrm{~Pa} \cdot \mathrm{s}$, 
研究材料黏度对生物凝胶挤出胀大的影响规律。从 图 4 所示的模拟结果中可以看出, 随着材料黏度的 增加, 凝胶纤维的挤出胀大率逐渐下降。这是由于 供料压力恒定时, 黏度增加导致流动阻力上升, 材 料的流动速度下降, 从而延长了其在针头内的停留 时间, 使得凝胶材料在挤出过程中得以释放出更多 的弹性能。但是, 随着黏度的变化, 凝胶挤出胀大 率的变化并不明显, 说明材料黏度并不是影响生物 凝胶挤出胀大的本质因素。

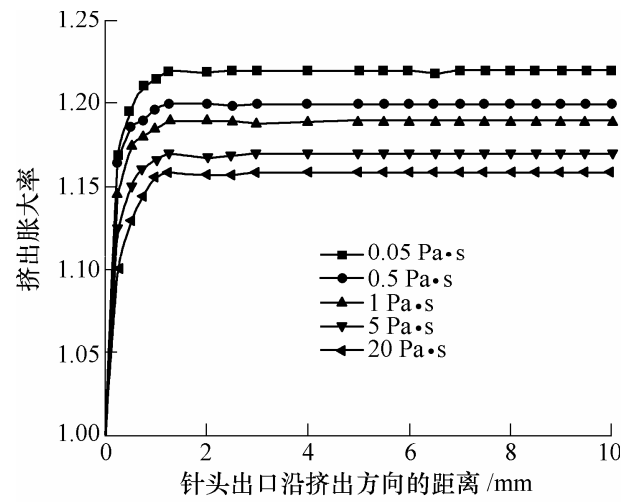

图 4 材料黏度对生物凝胶挤出胀大的影响

在支架实际成形过程中, 材料流速由供料压力 决定, 因此可以通过改变初始供料压力的大小, 研 究材料的挤出速度对生物凝胶挤出胀大的影响规 律。根据加工经验, 初始供料压力 $p_{0}$ 分别取为 0.3 $\mathrm{MPa} 、 0.4 \mathrm{MPa} 、 0.5 \mathrm{MPa} 、 0.6 \mathrm{MPa} 、 0.7 \mathrm{MPa}$ ，材料 黏度 $\eta=1 \mathrm{~Pa} \cdot \mathrm{S}$ 。图 5 为供料压力对凝胶挤出胀大的 影响。计算结果表明, 生物凝胶的挤出胀大率随着 供料压力的增加而发生明显的上升。这是由于凝胶 流体在喷头内的流动速度随着供料压力的上升而加 快, 相应缩短了拉伸弹性能在流道中的回复时间, 导致凝胶材料的挤出胀大率随之迅速增大。另外, 随着供料压力的增加, 挤出纤维的直径达到稳定时 距离针口的距离近似一致。

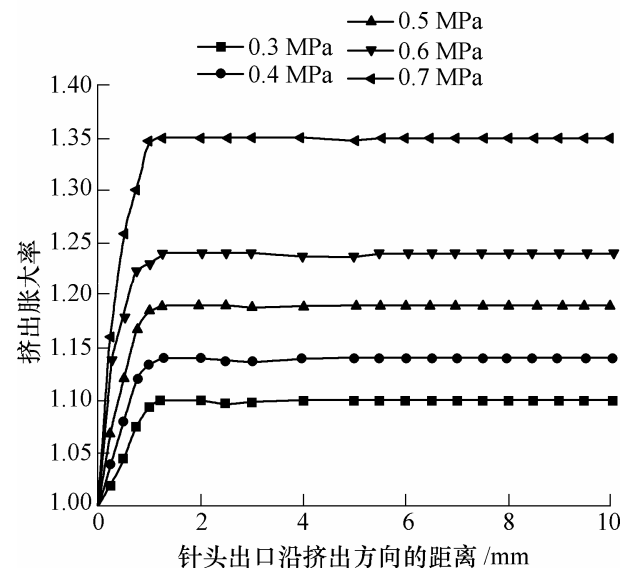

图 5 供料压力对生物凝胶挤出胀大的影响

\section{3 试验验证}

采用上海大学自主研发的组织工程支架三维 打印成形系统在 $25{ }^{\circ} \mathrm{C}$ 条件下进行凝胶纤维挤出成 形试验。选用质量分数分别为 $7 \%$ 的明胶溶液与 $3.8 \%$ 的海藻酸钠溶液等体积混合后搅拌均匀, 得到 生物凝胶材料; 采用气压供料方式, 挤出针头的内 径为 $0.51 \mathrm{~mm}$; 设定纤维接收平台的运动速度与材 料挤出速度相同，降低因拉伸或压缩对成形纤维形 态的影响。材料通过针头的挤出速度可按式(6)、(7) 进行计算 ${ }^{[15]}$

$$
\begin{gathered}
Q=\pi \frac{d^{3}}{8 K^{1 / n} \tau_{\mathrm{w}}^{3}}\left(\tau_{\mathrm{w}}-\tau_{0}\right)^{\frac{n+1}{n}}\left[\frac{n \tau_{\mathrm{w}}^{2}}{3 n+1}+\right. \\
\left.\frac{2 n^{2} \tau_{\mathrm{w}} \tau_{0}}{(2 n+1)(3 n+1)}+\frac{2 n^{3} \tau_{0}^{2}}{(n+1)(2 n+1)(3 n+1)}\right] \\
V=\frac{4 Q}{\pi d^{2}}
\end{gathered}
$$

式中 $K, n$ 一材料常数;

$\tau_{\mathrm{w},} \tau_{0}$ —流体在针头内流动时的切应力和滑 动应力;

$V$ 一一材料挤出时的流动速度。

\section{1 材料黏度对凝胶纤维挤出成形的影响}

通过控制材料在 $4{ }^{\circ} \mathrm{C}$ 环境下进行交联的时间 长短, 获得如图 6 所示具有不同黏度的生物凝胶材 料, 使用旋转流变仪(RS6000, 德国)测量的黏度值 在表 1 中列出, 供料压力固定为 $p_{0}=0.5 \mathrm{MPa}$ 。挤 出成形的凝胶纤维如图 7 所示, 测量得到的纤维直 径如表 2 所示。当黏度较低时，凝胶材料具有较好 的流动性, 挤出速度快, 但无法形成圆柱形纤维; 随着黏度的增加, 凝胶纤维的成形效果逐渐改善, 纤维直径逐渐减小并接近针头的内径尺寸，这与有 限元模拟结果相近; 当材料黏度大于 $2 \mathrm{~Pa} \cdot \mathrm{s}$ 时, 固 定的供料压力无法满足材料连续挤出所需的压力

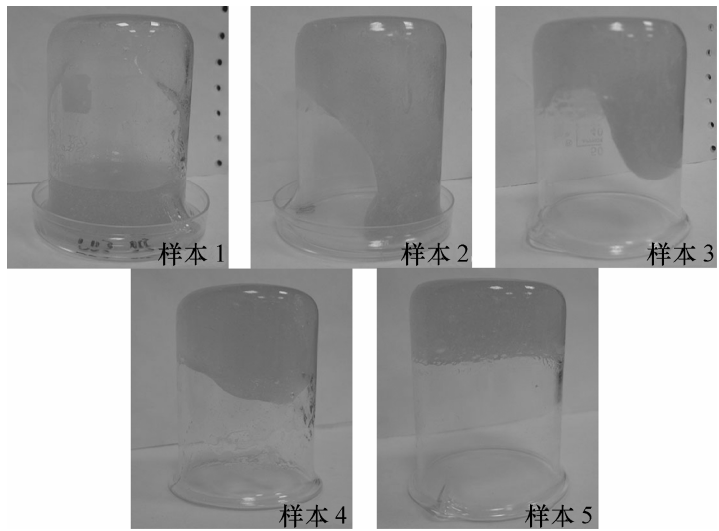

图 6 不同黏度的凝胶材料样本 
值, 只能形成断续的凝胶纤维, 直至无法挤出。试 验结果表明低黏度凝胶材料更易挤出, 但难以在常 温条件下成形; 更高的黏度能够保证纤维的成形效 果, 但挤出困难, 虽然挤出胀大作用减小, 会增加 因局部环流导致生物材料受热降解的可能。

表 1 明胶 + 海藻酸钠混合凝胶秥度测量值

\begin{tabular}{cccccc}
\hline 样本编号 & 1 & 2 & 3 & 4 & 5 \\
\hline 交联时间 $t / \min$ & 0 & 5 & 10 & 15 & 30 \\
黏度 $\eta /(\mathrm{Pa} \cdot \mathrm{s})$ & 0.100 & 0.466 & 1.217 & 3.285 & 22.052 \\
\hline
\end{tabular}

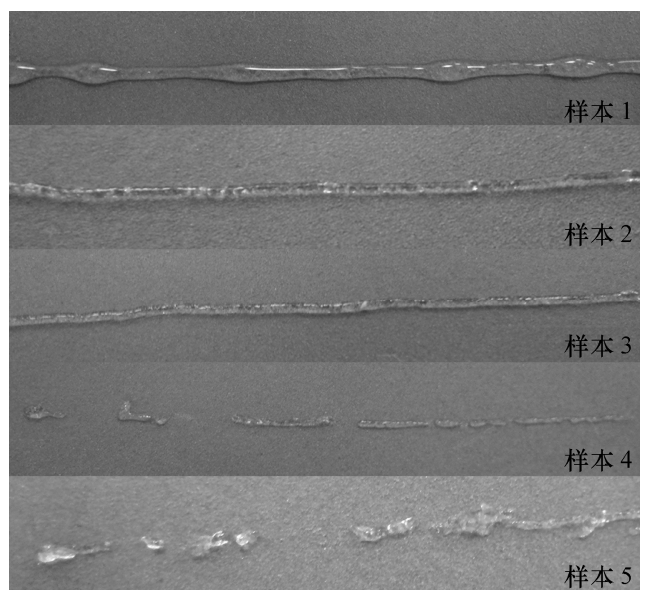

图 7 不同黏度的凝胶纤维成形效果

表 2 不同黏度下挤出纤维直径测量值

\begin{tabular}{cccccc}
\hline 样本编号 & 1 & 2 & 3 & 4 & 5 \\
\hline 直径 $d / \mathrm{mm}$ & - & 0.608 & 0.575 & 0.511 & - \\
\hline
\end{tabular}

\section{2 供料压力对凝胶纤维挤出成形的影响}

供料压力 $p_{0}$ 分别取为 $0.3 \mathrm{MPa} 、 0.4 \mathrm{MPa} 、 0.5$

$\mathrm{MPa} 、 0.6 \mathrm{MPa} 、 0.7 \mathrm{MPa}$ ，根据黏度对凝胶挤出胀 大和纤维成形的模拟、试验结果, 选择材料样本 3 (黏 度为 $1.217 \mathrm{~Pa} \cdot \mathrm{s}$ )进行凝胶纤维挤出成形试验, 结果 如图 8 所示。当供料压力小于 $0.5 \mathrm{MPa}$ 时, 材料体 积流量小于理想值, 纤维在成形过程中受到的拉伸 作用大于挤出胀大对纤维直径的影响, 因此直径小 于针头内径; 随着供料压力的增加, 材料的体积流 量上升, 挤出纤维的连续性逐渐改善; 同时, 由于 材料流动速度随供料压力的上升而加快, 挤出胀大

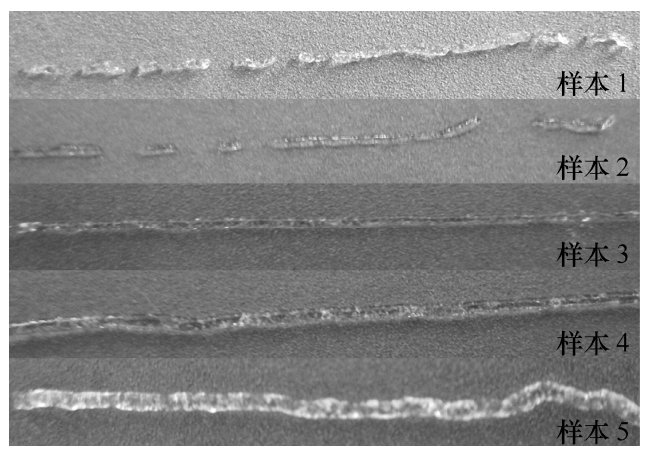

图 8 不同供料压力下凝胶纤维成形效果
作用导致纤维直径明显变粗, 测量的纤维直径如表 3 所示, 纤维挤出胀大随供料压力的变化规律与模 拟结果吻合良好。

表 3 不同压力下挤出纤维直径测量值

\begin{tabular}{cccccc}
\hline 样本编号 & 1 & 2 & 3 & 4 & 5 \\
\hline 直径 $d / \mathrm{mm}$ & 0.484 & 0.506 & 0.583 & 0.631 & 0.705 \\
\hline
\end{tabular}

\section{4 凝胶组织工程支架三维打印成形}

材料黏度是决定生物凝胶在常温条件下能否 打印成形并决定支架内部微观孔隙结构精度的关键 因素, 供料压力是影响生物凝胶挤出胀大的重要参 数。通过有限元模拟和试验验证, 选择最优的材料 属性和工艺参数, 以较低的供料压力、较小的挤出 胀大率和最佳的成形效果实现凝胶组织工程支架的 三维精确打印成形。

以微观孔隙尺寸为 $0.4 \mathrm{~mm}$, 孔隙率为 $60 \%$, 外 形尺寸为 $20 \mathrm{~mm} \times 20 \mathrm{~mm} \times 10 \mathrm{~mm}$ 的凝胶组织工程支 架为制备目标。通过模拟分析与试验结果的比较, 选择交联时间为 $10 \mathrm{~min}$ 的明胶与海藻酸钠混合制 备的生物凝胶为加工材料 $(\eta=1.217 \mathrm{~Pa} \cdot \mathrm{s})$, 供料压力 为 $0.53 \mathrm{MPa}( \pm 0.02 \mathrm{MPa})$, 针头内径为 $0.51 \mathrm{~mm}$ 。支 架孔隙率 $P$ 的计算公式

$$
P=\left(1-\frac{V_{\mathrm{s}}}{V_{\mathrm{z}}}\right) \times 100 \%
$$

式中 $V_{\mathrm{s}}$ 一支架材料的实体体积;

$V_{\mathrm{z}}$ 一支架外形的整体体积。

不考虑凝胶材料挤出胀大对成形支架微观孔 隙结构精度与孔隙率的影响, 输入如图 9 所示的目 标支架数字化模型进行支架制备。打印成形的凝胶 组织工程支架如图 10 所示, 支架纤维的平均直径为 $0.575 \mathrm{~mm}$, 与相似工艺条件下的有限元模拟结果 $0.618 \mathrm{~mm}$ 的误差率为 7\%, 造成这个误差的原因可 能是由于喷头的供料速度与支架材料接收平台的运 动速度之间存在匹配误差。成形支架的平均孔隙尺 寸为 $0.343 \mathrm{~mm}$, 与目标支架孔隙尺寸的相对误差为 $\Delta=(0.4-0.343) / 0.4 \times 100 \%=14.25 \%$; 孔隙率为 $49 \%$, 与目标支架的孔隙率要求相比下降了 $11 \%$ 。

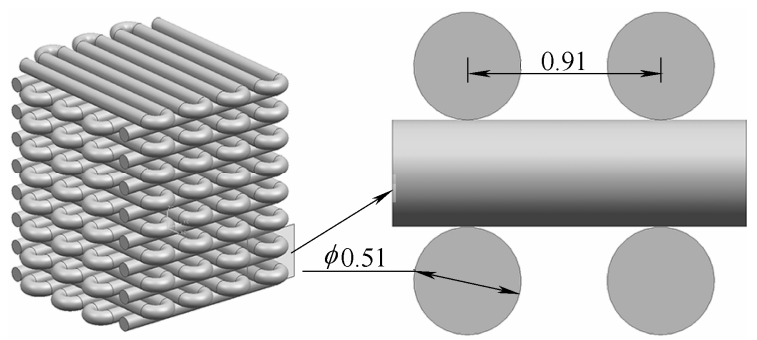

图 9 凝胶组织工程支架的原始数字化模型 


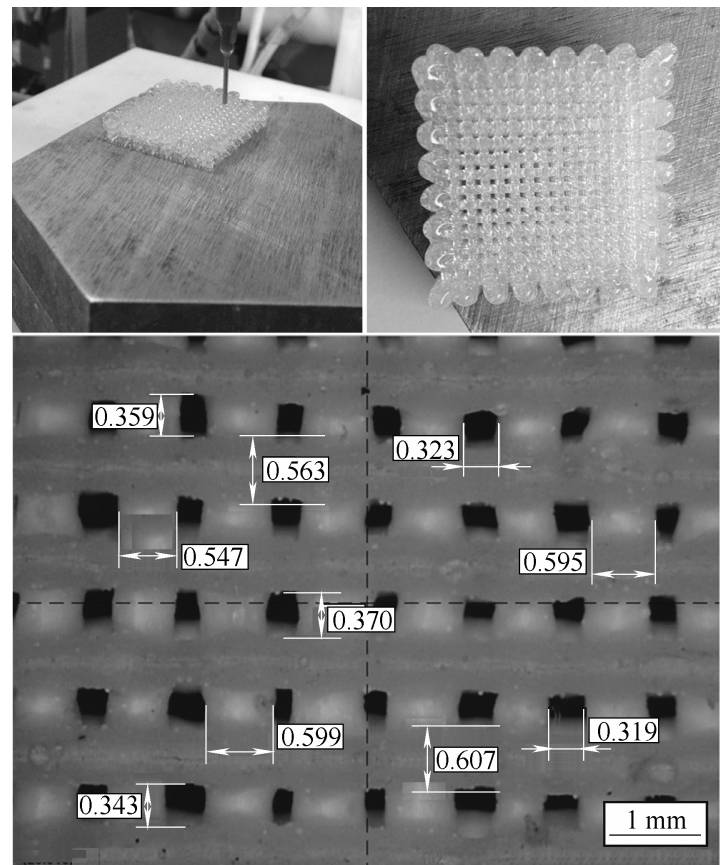

图 10 不考虑胀大影响的凝胶组织工程支架

考虑挤出胀大对支架微观孔隙尺寸与孔隙率 的影响, 将成形过程中由挤出胀大引起的纤维尺寸 误差引入支架的数字化模型, 增加模型中相邻纤维 间距, 设计如图 11 所示的修正模型。在相同的工艺 条件下进行凝胶组织工程支架的三维打印成形, 得 到如图 12 所示的凝胶支架。支架纤维的平均直径为 $0.571 \mathrm{~mm}$, 平均孔隙尺寸为 $0.413 \mathrm{~mm}$, 孔隙率为 $60.84 \%$, 成形支架的微观孔隙尺寸和孔隙率与目标 支架的孔隙结构要求高度吻合。

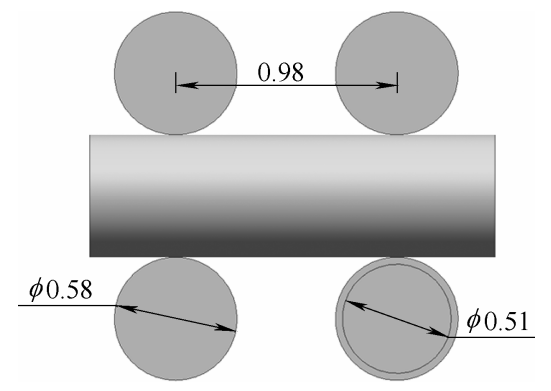

图 11 凝胶组织工程支架的修正数字化模型

由此可见, 通过对生物凝胶微孔挤出胀大的有 限元模拟可以揭示不同工艺参数对挤出胀大的影响 规律, 从而达到预测并优化支架模型与工艺参数的 目的, 最终实现凝胶组织工程支架在常温条件下的 精确打印成形, 可为进一步研究细胞与生物凝胶混 合的细胞三维精确受控组装成形奠定基础。

\section{5 结论}

（1）借助黏弹性 PTT 本构方程建立了描述生物

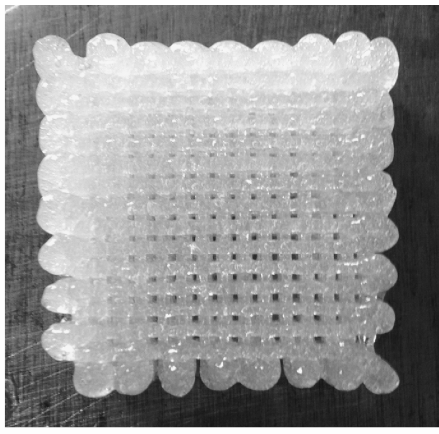

（a）考虑胀大影响的凝胶组织工程支架宏观结构

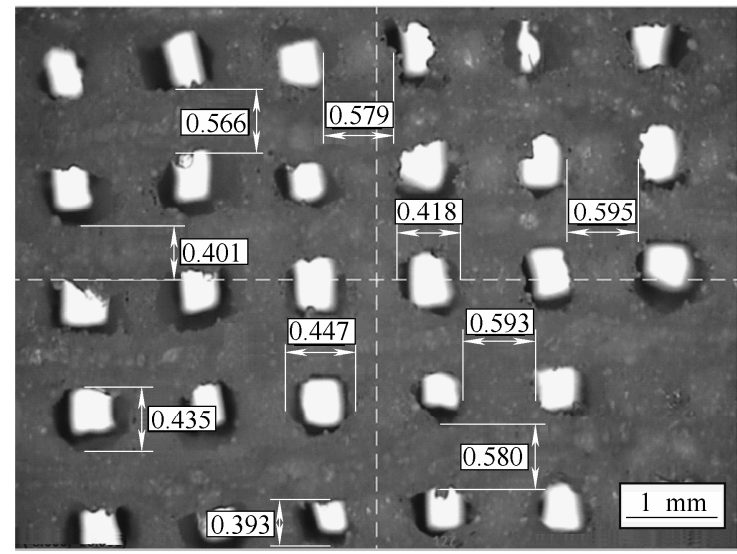

(b) 考虑胀大影响的凝胶组织工程支架孔隙结构

图 12 考虑胀大影响的凝胶组织工程支架

凝胶材料微孔挤出胀大过程的数学模型; 根据流道 直径的变化特点, 构建喷头直径过渡段和针头挤出 自由段的有限元模型, 通过参数渐近和网格重构的 方法模拟凝胶材料的挤出胀大过程。

（2）模拟结果表明，随着松弛时间的上升，凝 胶流体在喷头过渡段拐角处的局部环流现象逐渐加 剧; 供料压力恒定时, 凝胶材料的挤出胀大率随着 黏度的增大而减小; 材料黏度恒定时，凝胶材料的 挤出胀大率随着供料压力的上升而增大。通过凝胶 纤维的挤出成形试验, 验证了模拟的合理性。

（3）以制备微观孔隙尺寸为 $0.4 \mathrm{~mm}$, 孔隙率为 $60 \%$ 的支架为目标进行凝胶组织工程支架的三维打 印成形。不考虑凝胶材料挤出胀大的影响, 成形支 架的平均孔隙尺寸为 $0.343 \mathrm{~mm}$, 与目标支架的相对 误差 $\Delta=14.25 \%$, 孔隙率为 $49 \%$; 考虑挤出胀大的 影响, 调整模型中的纤维间距, 成形支架的平均孔 隙尺寸为 $0.413 \mathrm{~mm}$, 孔隙率为 $60.84 \%$, 与目标支 架的孔隙结构要求基本吻合。

（4）通过对生物凝胶微孔挤出胀大的有限元模 拟与凝胶组织工程支架的三维打印成形, 可以为进 一步研究细胞的三维精确受控组装成形提供理论参 考与试验依据。 


\section{参 考 文 献}

[1] LEONG K F, CHEAH C M, CHUA C K. Solid freeform fabrication of three-dimensional scaffolds for engineering replacement tissues and orgnas[J]. Biomaterials, 2003, 24(24): 2363-2378.

[2] FREYMAN T M, TANNAS I V, GIBSON L J. Cellular materials as porous scaffolds for tissue engineering $[\mathrm{J}]$. Progress in Materials Science, 2001，46(3-4): 273-282.

[3] 孟洁. 组织工程支架拓扑结构及化学成分对细胞行为 的影响[D]. 北京: 中国协和医科大学, 2007.

MENG Jie. Effect of topological structure and chemical constituents of tissue engineering scaffold on cell behavior[D]. Beijing: China Union Medical University, 2007.

[4] 尤飞, 姚远, 胡庆夕. 仿生骨支架微观孔结构的构建与 评价[J]. 机械工程学报, 2010, 46(2): 47-53.

YOU Fei, YAO Yuan, HU Qingxi. Generation and evaluation of porous structure of bionic bone scaffold[J]. Journal of Mechanical Engineering, 2010, 46(2): 47-53.

[5] VOZZI G, PREVITI A, ROSSI D, et al. Microsyringe based deposition of two-dimensional and three-dimensional polymer scaffolds with a well-defined geometry for applications to tissue engineering[J]. Tissue Engineering, 2002, 8(6): 1089-1098.

[6] WOODFIELD T B F, MALDA J, WIJN J, et al. Design of porous scaffolds for cartilage tissue engineering using a three-dimensional fibre-deposition technique[J]. Biomaterials, 2004, 25(18): 4149-4161.

[7] KHALIL S, SU W. Biopolymer deposition for freeform fabrication of hydrogel tissue constructs[J]. Materials Science and Engineering: C, 2007, C27: 469-478.

[8] KE Hui. Investigation into the dispensing-based fabrication process for tissue scaffolds[D]. Saskatoon: University of Saskatchewan, 2006.

[9] ALEXE-LONESCU A L, BARBERO G, MEYER C. Influence of the rheological properties on the electrical impedance of hydrogels[J]. Journal of Applied Physics, 2012, 111: 014905.

[10] GLASSMAN M J, CHAN J, OLSEN B D. Reinforcement of shear thinning protein hydrogels by responsive block copolymer self-assembly[J]. Advanced Functional Materials, 2013, 23(9): 1182-1193.

[11] LI Minggan, TIAN Xiaoyu, CHEN Xiongbiao. Modeling of flow rate, pore size, and porosity for the dispensing-based tissue scaffolds fabrication[J]. Journal of Manufacturing Science and Engineering, 2009, 131: 034501.

[12] 李生杰, 颜永年, 王小红, 等. 多喷头细胞受控组装过 程的启停响应控制[J]. 电加工与模具, 2010(1): 9-12, 16. LI Shengjie, YAN Yongnian, WANG Xiaohong, et al. Nozzle-opening/closing response control on multi-nozzle cell controlled assembling process[J]. Electromachining \& Mould, 2010(1): 9-12, 16.

[13] 杨敏, 徐铭恩, 陈洁毅, 等. 基于气动控制的多细胞三 维组装技术研究[J]. 中国生物医学工程学报, 2013, 32(2): 211-219.

YANG Min, XU Mingen, CHEN Jieyi, et al. Research on three-dimension multi-cell assembly technique based on pneumatic control[J]. Chinese Journal of Biomedical Engineering, 2013, 32(2): 211-219.

[14] 戴元坎. 汽车橡胶密封条挤出成型过程的计算机模拟 研究[D]. 上海: 上海交通大学, 2008 .

DAI Yuankan. Study of the extrusion simulation of auto sealing strip of rubber[D]. Shanghai: Shanghai Jiao Tong University, 2008.

[15] CHEN Xiongbiao, LI Minggan, KE Hui. Modeling of the flow rate in the dispensing-based process for fabricating tissue scaffolds[J]. Journal of Manufacturing Science and Engineering, 2008, 130: 021003.

[16] 夏杨. 海藻酸钠-明胶共混体系为载体的可注射式组织 工程骨的研究[D]. 北京: 中国协和医科大学, 2008 .

XIA Yang. Study on injectable tissue engineering bone with align-gelatin complex as carriers[D]. Beijing: China Union Medical University, 2008.

作者简介: 于永泽, 男, 1984 年出生, 博士研究生。主要研究方向为快 速成形工艺与再生组织工程支架制备。

E-mail: yuyz1023@sina.com

胡庆夕(通信作者), 男, 1959 年出生, 博士, 研究员, 博士研究生导师。 主要研究方向为快速成形、仿生建模与再生骨支架制造。

E-mail: huqingxi@shu.edu.cn 